\title{
Disorder effects on the drag rate in double-quantum-wire systems
}

\author{
B. Tanatar \\ Department of Physics, Bilkent University, Bilkent, 06533 Ankara, Turkey
}

(Received 6 October 1997; revised manuscript received 17 February 1998)

\begin{abstract}
We study the Coulomb drag rate for electrons in a double-quantum-wire structure in the presence of disorder. The particle-number-conserving relaxation-time approximation is used to phenomenologically broaden the response functions in the drag rate to account for the disorder effects. The interplay between the screening effects and disorder at high temperature yields a nonmonotone behavior of the drag rate on the disorder parameter. The reduction in the interwire momentum transfer rate may be used as a probe to investigate localization properties of coupled quantum wires. [S0163-1829(98)04427-0]
\end{abstract}

Coupled quantum-well systems are especially well suited to probe many-body effects because of the interplay between the in-layer and across the layer interaction strengths. An example is the Coulomb drag effect, when the well separation is large enough so that tunneling effects are not important; a current flowing in one layer induces a current or voltage in the other layer. ${ }^{1}$ The origin of the effect is the interactions between the electrons in different layers leading to momentum and energy transfer from the current-carrying layer to the passive one. The experiments ${ }^{2-5}$ performed at low temperature gave way to a surge of theoretical activity ${ }^{6-10}$ to understand the transport properties of spatially separated electronic systems. The high-temperature behavior of the Coulomb drag rate was explained ${ }^{8}$ by the collective mode effects influencing the effective interlayer interaction and leading to an enhancement, which has recently been observed experimentally. ${ }^{11}$ The quasi-one-dimensional (1D) semiconductor structures provide another example to study the momentum and energy transfer between two electron gases of close proximity. The Coulomb drag effect for quantum-wire systems was considered by Sirenko and Vasilopoulos ${ }^{12}$ in their comparative study of dimensionality effects. Qin ${ }^{13}$ used a cylindrical confinement model to determine the temperature and wire radius dependence of the momentum transfer rate. There has been no drag measurements on quantum wires to date.

In this paper, we study the effects of disorder on the Coulomb drag rate in coupled quantum wires in the plasmondominated high-temperature region. There are several motivations for investigating the disorder effects. The interplay between the electron-electron interactions and disorder has been a subject of great interest accentuated with the recent observation of metal-insulator transition. ${ }^{14}$ The Coulomb drag effect in double-layer and double-wire systems offers an interesting probe in diagnosing the insulating phase as suggested by Shimshoni. ${ }^{15}$ Since the drag rate is predicted to be enhanced by the plasmon modes, the disorder effects may be more easily discerned at higher temperatures than the lowtemperature region where virtual phonon exchange mechanism also influences the observed behavior. The effects of optical phonons are argued to be weak ${ }^{16}$ and we do not consider them.

Since the impurity effects are expected to influence the transport properties, in the typical experiments high-mobility samples are used. The Coulomb drag contribution to the observed momentum-transfer rate is then calculated with the assumption that intralayer impurity scattering is small and independent of energy. ${ }^{8,9}$ Flensberg et al. ${ }^{9}$ discussed the necessary modifications to the drag resistivity in the case of energy-dependent electron-impurity scattering. Recently, Świerkowski et al. ${ }^{17}$ presented a linear-response theory for transresistance in double-layer semiconductor structures. In their treatment disorder scattering through the relaxation time approximation is accounted for. Our aim is to study the effects of disorder on the Coulomb drag rate at high temperature. We calculate the interwire momentum transfer rate for a coupled quantum-wire system by systematically increasing the strength of disorder parameter, which amounts to decreasing the mobilities in each wire and can be achieved experimentally by using disordered samples in a systematic way. We find that the interplay between the disorder effects and effective electron-electron interactions gives rise to an increase in the drag rate for small values of the disorder parameter. As the strength of disorder is further increased we find that the drag rate decreases.

We consider two parallel cylindrical quantum wires of radius $R$ and infinite potential barriers. ${ }^{18}$ The axes of the wires are separated by a distance $d$, which is large enough to prevent interlayer tunneling. The bare Coulomb interaction between the electrons is written as $V_{i j}(q)=\left(2 e^{2} / \epsilon_{0}\right) F_{i j}(q)$, in which the form factors $F_{i j}(q)$ describe the intra and interwire interactions. ${ }^{18}$ The one-dimensional electron density $N$ is related to the Fermi wave vector by $N=2 k_{F} / \pi$. We also define the dimensionless electron gas parameter $r_{s}$ $=\pi /\left(4 k_{F} a_{B}^{*}\right)$, in which $a_{B}^{*}=\epsilon_{0} /\left(e^{2} m^{*}\right)$ is the effective Bohr radius in the semiconducting layer with background dielectric constant $\epsilon_{0}$ and electron effective mass $m^{*}$.

We adopt the Coulomb drag-rate expression derived for double-layer systems to the present case of double-wire problem, ${ }^{6-10}$

$$
\tau_{D}^{-1}=\frac{1}{4 \pi m^{*} N T} \int_{0}^{\infty} d q q^{2} \int_{0}^{\infty} d \omega\left|\frac{W_{12} \operatorname{Im} \chi(q, \omega)}{\sinh (\omega / 2 T)}\right|^{2},
$$

in which we have assumed equal density quantum wires (we set $\hbar$ and $\left.k_{B}=1\right) . \tau_{D}^{-1}$ measures the rate of momentum transferred from one quantum wire to the other. Here, $\chi(q, \omega)$ is the 1D dynamic susceptibility, describing the density-density 
response function of a single wire. $W_{12}(q, \omega)$ is the dynamically screened effective interaction between electrons in quantum wires 1 and 2. Within the random-phase approximation (RPA), it is given by $W_{12}(q, \omega)=V_{12}(q) / \varepsilon(q, \omega)$, where $\varepsilon(q, \omega)=\left[1-V_{11}(q) \chi(q, \omega)\right]^{2}-\left[V_{12}(q) \chi(q, \omega)\right]^{2}$ is the total screening function for the coupled quantum-wire system that uses the bare intrawire and interwire electronelectron interactions ignoring the correlation effects. Recent numerical calculations ${ }^{19}$ indicate the importance of correlations in coupled quantum-wire systems. It is also assumed that only the lowest subband in each wire is occupied. Thus, the energy difference between the second and first subband levels $\Delta_{21} \approx 10(4 / \pi)^{2} r_{s}^{2}\left(R / a_{B}^{*}\right) E_{F}$ should be greater than the thermal energy $T$. For densities and wire radii of experimental interest, the single subband assumption holds.

In this work, we retain the full wave vector, frequency, disorder, and temperature dependence of the dynamic susceptibility $\chi(q, \omega)$ that enters the drag-rate expression [Eq. (1)] as well as the screening function $\varepsilon(q, \omega)$. We account for disorder by considering an impurity scattering induced broadening $\gamma$ that is a phenomenological parameter within the number-conserving approximation given by

$$
\chi_{\gamma}(q, \omega)=\frac{(\omega+i \gamma) \chi(q, \omega+i \gamma)}{\omega+i \gamma \chi(q, \omega+i \gamma) / \chi(q, 0)} .
$$

Thus, in the drag-rate integral we use the above polarizability expression that includes both the temperature and impurity scattering effects. In the limit $q, \omega \rightarrow 0$, the numberconserving approximation above gives the correct diffusive behavior for the response function $\chi_{\gamma}(q, \omega) \simeq$ $-\left(2 m^{*} / \pi k_{F}\right)\left(D q^{2} / D q^{2}+i \omega\right)$, where $D=k_{F}^{2} / m^{* 2} \gamma$ is the diffusion constant in a $1 \mathrm{D}$ system.

We use parameters appropriate for a GaAs system for which the recent experiments ${ }^{2-5,11}$ on the drag rate between coupled quantum wells are performed, and first examine $\tau_{D}^{-1}$ at low temperatures. In coupled quantum-wire systems with a single filled subband, $\tau_{D}^{-1}$ is dominated by backscattering $\left(q \sim 2 k_{F}\right)$. At low temperatures $\left(T \ll T_{F}\right)$, the use of approximate expressions for the response function $\chi(q, \omega)$ of a clean system, and neglecting the screening effects result in a linear temperature dependence ${ }^{12} \tau_{D}^{-1} \sim\left|W_{12}\left(2 k_{F}\right)\right|^{2}\left(m^{* 2} T / k_{F}^{2}\right)$. In the presence of disorder, the diffusive limit of $\chi(q, \omega)$ gives rise to a different $\omega$ and $q$ behavior, and we find to leading order $\tau_{D}^{-1} \sim\left|W_{12}\left(2 k_{F}\right)\right|^{2}\left(m^{* 5} T^{2} \gamma^{2} / k_{F}^{8}\right)$. In two-dimensional systems, Zheng and MacDonald ${ }^{6}$ using similar approximations have found a logarithmic correction to the lowtemperature drag rate. Kamenev and $\mathrm{Oreg}^{9}$ have also reached similar results, and in particular have shown that for extremely dirty samples the drag resistivity goes as $\rho_{D} \sim T \gamma$.

Next, we evaluate $\tau_{D}^{-1}$ numerically using the effective interaction obtained for a double-wire system as a function of temperature. Similar to the double-quantum-well system, ${ }^{8,11}$ at high temperature the drag rate is dominated by collective excitation modes described by the zeros of the dielectric function $\varepsilon(q, \omega)$. The plasmon dispersion $\omega_{\mathrm{pl}}(q)$ in a double-wire system has two branches, both lying above the particle-hole continuum. As the temperature increases, the particle-hole continuum embodying the single-particle excitation region broadens to render coupling between the collective modes more feasible, and $\tau_{D}^{-1}$ is enhanced. The

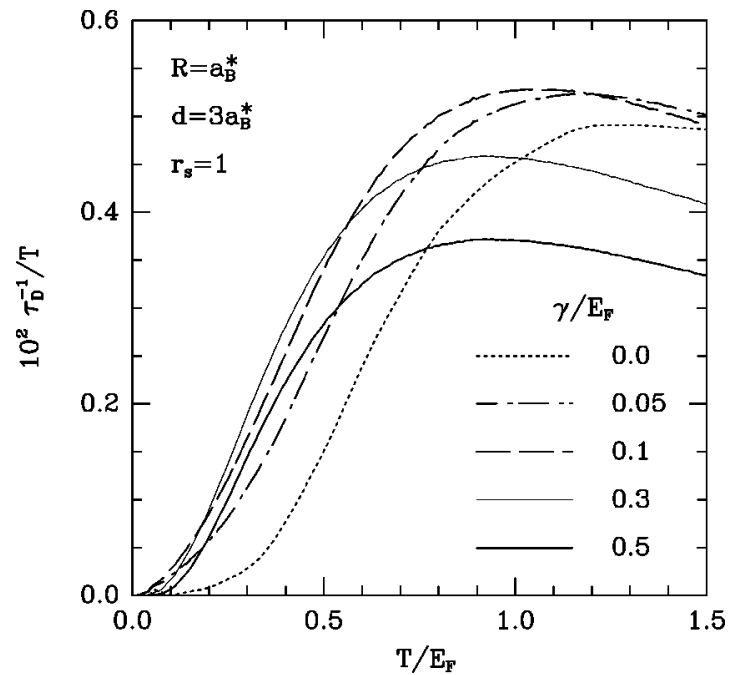

FIG. 1. The scaled drag rate $\tau_{D}^{-1} / T$ within the RPA as a function of temperature for a double-quantum-wire system.

effect of broadening to simulate disorder effects on the plasmon dispersions is such that $\omega_{\mathrm{pl}}(q)$ is depressed. In Fig. 1 we show the scaled drag rate $\tau_{D}^{-1} / T$ as a function of temperature for a typical coupled-wire system. That $\tau_{D}^{-1} / T$ exhibits a broad enhancement for $T \gtrsim 0.3 E_{F}$ indicates a strong $T$ dependence at high temperature. We observe that with increasing disorder (for small $\gamma$ ) $\tau_{D}^{-1}$ increases in magnitude and shifts towards the low-temperature side. Further increase in $\gamma$ results in a decrease in the drag rate for large temperatures. At the largest disorder parameter considered $\left(\gamma / E_{F}\right.$ $=0.5)$ the drag rate is considerably lower than that of a clean system $(\gamma=0)$.

To trace the origin of dependence on the disorder parameter $\gamma$ of the momentum transfer rate we investigate the integrand of Eq. (1) in detail. After performing the frequency integral, we end up with $\tau_{D}^{-1} \sim \int d q q^{2} F(q)$, which we plot in Fig. 2 as a function of $q$. Specializing to the coupled-wire system with parameters $R=a_{B}^{*}$ and $d=3 a_{B}^{*}$, at $r_{s}=1$ and $T=E_{F}$, we observe that the peak position in the integrand is shifted towards the long-wavelength side as $\gamma$ increases. However, the peak height of the integrand after increasing for low disorder $\left(\gamma \approx 0.1 E_{F}\right)$ starts to decease for greater disorder compared to its value of the clean system. Figure $3(a)$ shows $\operatorname{Im}[\chi(q, \omega)]$ and the dynamically screened response function $\operatorname{Im}[\chi(q, \omega) /|\varepsilon(q, \omega)|]$ as a function of frequency. We observe a steady decrease as the disorder parameter $\gamma$ increases. However, at a smaller wave vector $(q$ $\left.=0.1 k_{F}\right)$ we find in Fig. 3(b) a rather different behavior for the screened quantity $\operatorname{Im}[\chi(q, \omega)] /|\varepsilon(q, \omega)|$. As the integral over $q$ and $\omega$ is performed in the calculation of $\tau_{D}^{-1}$ the observed nonmonotone behavior ensues.

We have based our systematic study of disorder scattering effects on $\tau_{D}^{-1}$ on the theoretical formalism developed by Swierkowski et al. ${ }^{17}$ In this approach momentumindependent relaxation-time approximation is used to phenomenologically broaden the response function $\chi(q, \omega)$. A number of calculations are devoted to the low-temperature behavior of drag rate for coupled quantum wells in the presence of disorder. By splitting the contributions of ballistic and diffusive regimes Zheng and MacDonald ${ }^{6}$ calculated the 


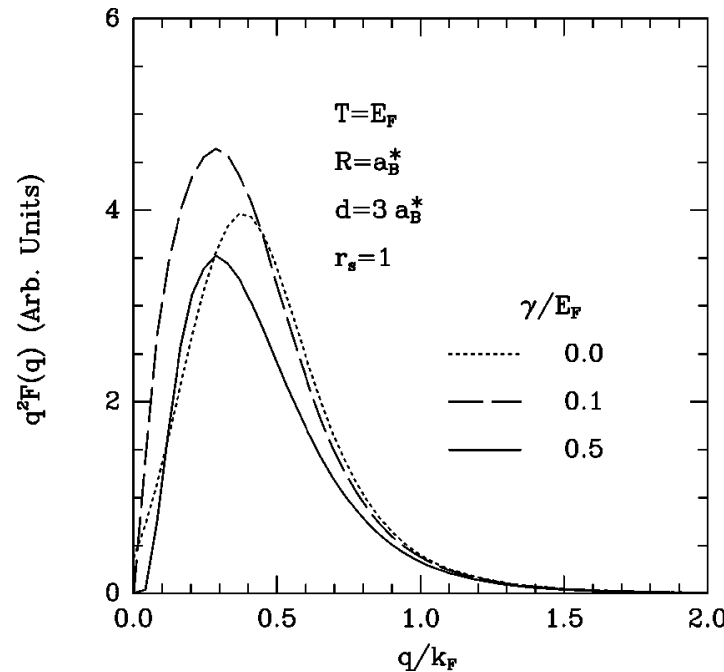

FIG. 2. The integrand of Eq. (1) after the $\omega$ integration is carried out.
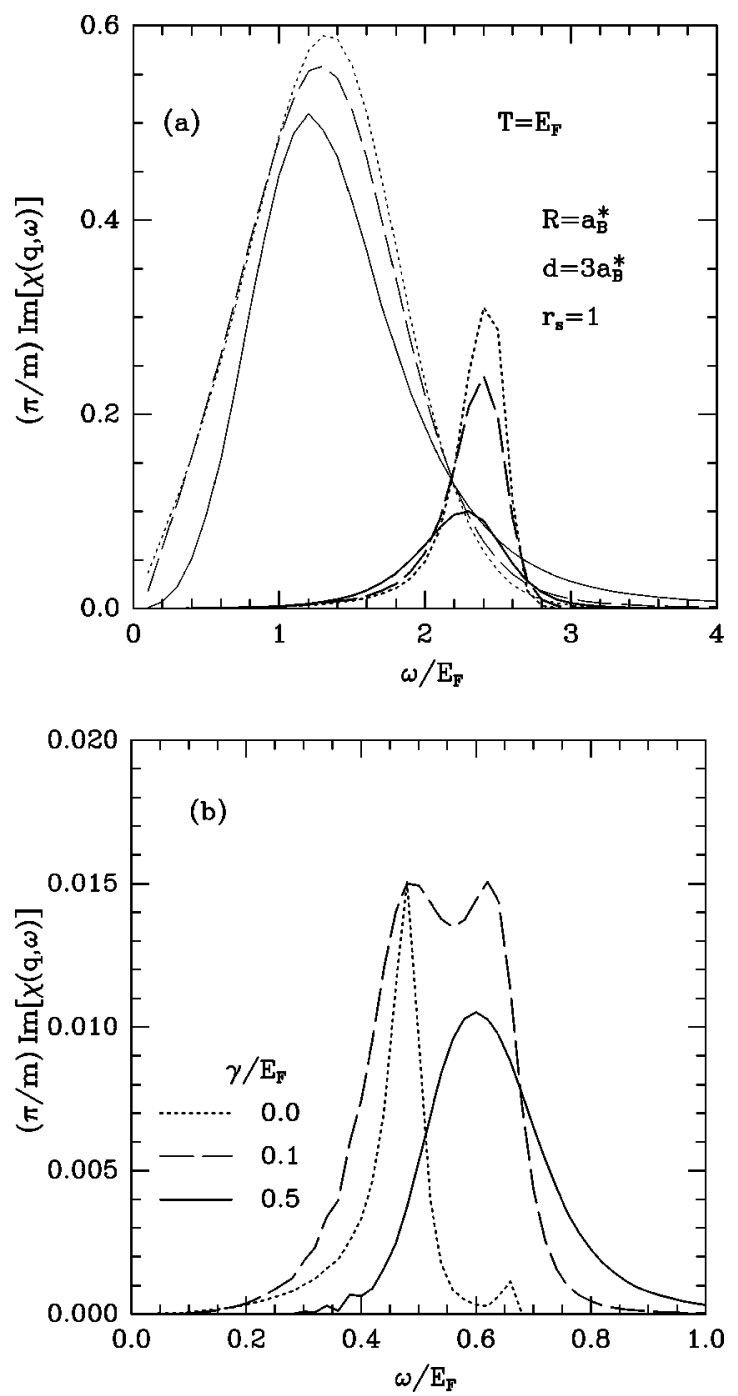

FIG. 3. The frequency dependence of $\operatorname{Im}[\chi(q, \omega)]$. (a) Thin lines are for the noninteracting system, whereas the thick lines denote $\operatorname{Im}[\chi(q, \omega)] /|\varepsilon(q, \omega)|$ at $q=0.5 k_{F}$. (b) $\operatorname{Im}[\chi(q, \omega)] /|\varepsilon(q, \omega)|$ for the same parameters at $q=0.1 k_{F}$.
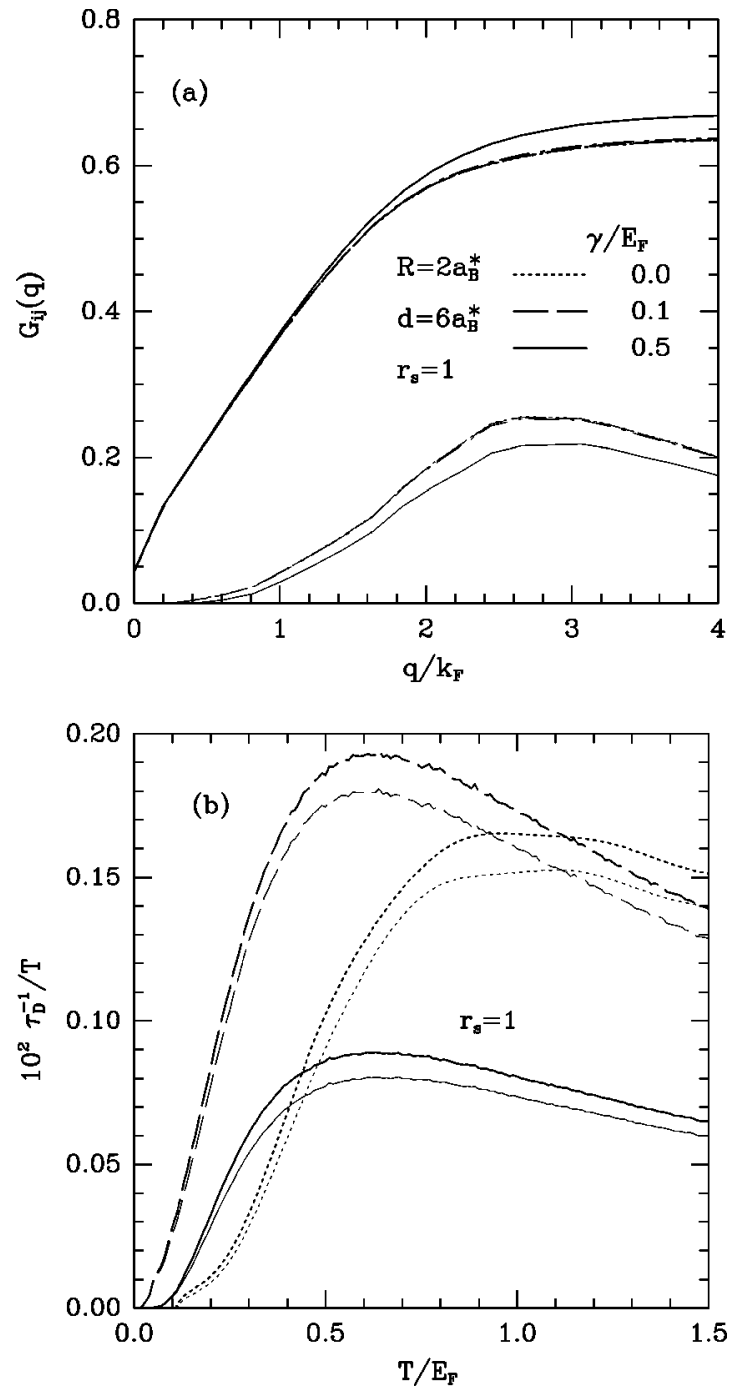

FIG. 4. (a) The intrawire (thick lines) and interwire (thin lines) local-field corrections in the presence of disorder in a coupled quantum-wire system. The interwire local-field factors are multiplied by a factor of 10 . (b) $\tau_{D}^{-1} / T$ with (thick lines) and without (thin lines, RPA) the local-field corrections.

correction to the interlayer scattering rate due to disorderenhanced interactions. Similar enhancement in the drag resistivity was also calculated by Kamenev and $\mathrm{Oreg}^{9}$ who used diagrammatic perturbation theory. In a recent paper, Shimshoni ${ }^{15}$ considered the Coulomb drag between two parallel layers in the Anderson insulating state, treating the Mott and Efros-Shklovskii types separately. In his lowtemperature analysis, Shimshoni ${ }^{15}$ found that $\rho_{D}$ is suppressed for a Mott insulator with decreasing localization length (i.e., increasing disorder). In all these attempts the disorder has the effect of enhancing $\tau_{D}^{-1}$ of the resistivity $\rho_{D}$ as a function of $T$. In the Boltzmann theory-based calculation of the drag rate Flensberg and $\mathrm{Hu}^{8}$ found that the charged impurities located a distance $s$ away from the quantum wells influenced $\tau_{D}^{-1}$ significantly for $s \lesssim 400 \AA$. Classical simulations to determine the influence of ionized impurities on Coulomb drag have also been performed. ${ }^{20} \mathrm{We}$ also point out that disorder effects in Coulomb drag problems are gaining attention recently in a variety of related contexts. ${ }^{21}$ Our approach is different than considered by Shimshoni ${ }^{15}$ in that we 
assume from the outset that the electronic state of quantum wires are metallic. The phenomenological disorder parameter has the effect of lowering the mean free path of electrons as the magnitude of $\gamma$ increases. Thus, the density fluctuations described by $\operatorname{Im}[\chi(q, \omega)]$ in the numerator of Eq. (1), and $\varepsilon(q, \omega)$ appearing in the denominator are nontrivially altered at higher temperatures. Taking the mean free path and localization length in a 1D system to be the same, we estimate $l k_{F}=2 E_{F} / \gamma \approx 7$ for $\gamma / E_{F} \approx 0.3$, which is close to the weak to strong localization crossover. Our results suggest that coming from the metallic phase, the drag rate may potentially signal the localization properties of coupled quantumwire systems.

As the electron density is lowered the exchangecorrelation effects become stronger, rendering the RPA inadequate. In the studies of drag resistivity and drag rate in double-layer systems it has been found important to include correlation effects beyond those described by the RPA to achieve agreement with experimental data at low densities. ${ }^{16,22} \mathrm{We}$ incorporate the correlation effects through the local-field corrections calculated within the selfconsistent field method, ${ }^{19}$ which also includes the disorder effects. In this number-conserving approximation with $\gamma$ acting as a parameter throughout the self-consistent evaluation of the correlation effects, we find that the local-field factors are slightly modified. Figure 4(a) shows the intrawire (thick lines) $G_{11}$ and interwire (thin lines) $G_{12}$ local-field corrections for a coupled quantum-wire system. It is found that the phenomenological disorder parameter $\gamma$ changes $G_{i j}(q)$ for $q / k_{F} \gtrsim 1$, with the general effect of increasing the intrawire correlations and decreasing the interwire correlations. A calculation by Swierkowski et al. ${ }^{17}$ shows that the interlayer local-field factor $G_{12}$ affects the transresistivity in doublelayer electron systems very little. However, the short-range intralayer correlations built in via the self-consistent scheme yield a substantial increase. In Fig. 4(b) we show $\tau_{D}^{-1}$ with (thick lines) and without (thin lines, RPA) the local-field corrections for $r_{s}=1$, and observe that the correlation effects increase the calculated drag rate.

In summary, we have considered the Coulomb drag effect between two parallel quantum wires in the presence of disorder treated phenomenologically. The temperature dependence of the drag rate is known to be significantly enhanced at high temperature when a dynamically screened effective interlayer interaction is used due to the collective density fluctuations in the double-quantum-wire system. We find that for small $\gamma$, the drag rate is further increased. For larger values of $\gamma$, the density fluctuations are suppressed with a reduced localization length and the drag rate is reduced. Thus, the drag rate $\tau_{D}^{-1}$ exhibits a nonmonotonous behavior with respect to the strength of disorder, and may be used as a possible probe to understand the localization properties in Coulomb-coupled systems. A systematic experimental study with varying degrees of disorder should, in principle, be able to test some of our predictions.

This work was partially supported by the Scientific and Technical Research Council of Turkey (TUBITAK). We thank Dr. M. Z. Gedik for fruitful discussions.
${ }^{1}$ M. B. Pogrebinskii, Fiz. Tekh. Poluprovodn. 11, 637 (1977)

[Sov. Phys. Semicond. 11, 372 (1977)]; P. J. Price, Physica B \& C 117, 750 (1983).

${ }^{2}$ P. M. Solomon, P. J. Price, D. J. Frank, and D. C. La Tulipe, Phys. Rev. Lett. 63, 2508 (1989).

${ }^{3}$ T. J. Gramila et al., Phys. Rev. Lett. 66, 1216 (1991); Phys. Rev. B 47, 12957 (1993).

${ }^{4}$ U. Sivan, P. M. Solomon, and H. Shtrikman, Phys. Rev. Lett. 68, 1196 (1992).

${ }^{5}$ H. Rubel et al., Semicond. Sci. Technol. 10, 1229 (1995).

${ }^{6}$ L. Zheng and A. H. MacDonald, Phys. Rev. B 48, 8203 (1993); A.-P. Jauho and H. Smith, ibid. 47, 4420 (1993).

${ }^{7}$ H. C. Tso, P. Vasilopoulos, and F. M. Peeters, Phys. Rev. Lett. 68, 2516 (1992); 70, 2146 (1993).

${ }^{8}$ K. Flensberg and B. Y.-K. Hu, Phys. Rev. Lett. 73, 3572 (1994); Phys. Rev. B 52, 14796 (1995).

${ }^{9}$ A. Kamenev and Y. Oreg, Phys. Rev. B 52, 7516 (1995); K. Flensberg et al., ibid. 52, 14761 (1995).

${ }^{10}$ B. Laikhtman and P. M. Solomon, Phys. Rev. B 41, 9921 (1990); I. I. Boiko and Yu. M. Sirenko, Phys. Status Solidi B 159, 805
(1990).

${ }^{11}$ N. P. R. Hill et al., Phys. Rev. Lett. 78, 2204 (1997).

${ }^{12}$ Yu. M. Sirenko and P. Vasilopoulos, Phys. Rev. B 46, 1611 (1992).

${ }^{13}$ G. Qin, J. Phys.: Condens. Matter 7, 9785 (1995).

${ }^{14}$ S. V. Kravchenko et al., Phys. Rev. B 50, 8039 (1994).

${ }^{15}$ E. Shimshoni, Phys. Rev. B 56, 13301 (1997).

${ }^{16}$ B. Y.-K. Hu, Phys. Rev. B 57, 12345 (1998).

${ }^{17}$ L. Świerkowski, J. Szymański, and Z. W. Gortel, Phys. Rev. B 55, 2280 (1997).

${ }^{18}$ A. Gold and A. Ghazali, Phys. Rev. B 41, 7626 (1990); A. Gold, Philos. Mag. Lett. 66, 163 (1992).

${ }^{19}$ N. Mutluay and B. Tanatar, Phys. Rev. B 55, 6697 (1997).

${ }^{20}$ V. Cambel and M. Moško, Semicond. Sci. Technol. 8, 364 (1993).

${ }^{21}$ Y. Oreg and A. Kamenev, Phys. Rev. Lett. 80, 2421 (1998); A. Stern and I. Ussishkin, Physica E 1, 176 (1997).

${ }^{22}$ L. Świerkowski, J. Szymański, and Z. W. Gortel, Phys. Rev. Lett. 74, 3245 (1995). 